\title{
On the Construction of Radio Environment Map for Cognitive Radio Applications: An Overview
}

\author{
Bara'u Gafai Najashi ${ }^{1}$ \\ Lecturer, Department of Electrical \& Computer Engineering, Baze University, Abuja, Nigeria ${ }^{1}$
}

\begin{abstract}
Demand for data services from a few Kilobytes to several Gigabytes over the last 25 years has led to the development of several mobile and wireless standards. Unfortunately, the spectrum required for these services to operate is becoming scarce not because of shortage but because of under-utilization as several spectrum occupancy measurements have shown over the years. The solution then has to be a dynamic spectrum access approach where licensed and unlicensed users could share the spectrum without causing interference to the licensed user. This concept known as Cognitive Radio (CR) was envisioned by Mitola promises to solve this problem. Radio Environment Map (REM) is one of the central tools at the heart of CR as it constructs a comprehensive map of the CR network storing information on physical network, policies, regulation, licensed user profile and activity. In this research, a general overview on REM construction is presented. From the architecture to the techniques applied in constructing REMs. The performance of propagation models employed in this process is also presented. Finally the quality metrics used to determine the accuracy of the constructed REMs is given
\end{abstract}

Keywords: Cognitive radio, Radio environment map, propagation models, quality metrics

\section{INTRODUCTION}

Electromagnetic Spectrum being the medium for wireless communication has of recent faced substantial paradigm shift. The increasing demand for new applications and services that are "bandwidth hungry" has led to developments of several concepts within the research community [1]. One of such concepts is cognitive radio which promises to revolutionize the mode of spectrum allocation from command and control to a dynamic approach where spectrum could be shared both spatially and temporally [2]. Successful deployment of cognitive radio requires mobile terminal/nodes to be aware of their environment so as to make informed and intelligent decisions without causing interference. To achieve this goal, mobile network nodes must be able to sense the channel to determine the presence or absence of a user [3].

The cognitive radio ecosystem therefore requires the sharing of network resources between licensed (primary) users and unlicensed (secondary) ones without causing interference to the primary user through sensing. It has therefore become necessary to develop models that could correctly predict the status of radio channels for initiation/termination of transmission by a mobile terminal. Spectrum occupancy measurements (radio measurements) have been performed over the years to determine the level of spectrum utilization and in extension utilize the data to develop models for spectrum hole prediction [4-6]. Radio measurements have become more efficient due to improvements measurement technologies coupled with recent innovations in data mining, efficient exploitation methods have been developed that make it possible to extract vital information to enhance the viability of new concepts such as Cognitive Radio (CR) [7].

According to Zhao in [8] who introduced and developed Radio Environment Maps (REM), he stated that "The REM can be viewed as an integrated database that provides multi-domain environmental information and prior knowledge for cognitive radios, such as the geographical features, available services and networks, spectral regulations, locations and activities of neighboring radios, policies of the users and/or service providers, and past experience." From the definition, it can be deduced that REM should contain information about the radio environment, the location of transmitters, possible areas of interference with or by other transmitters. REM's are therefore maps of Radio frequency (RF) power levels over a given geographical area that could be used for better spectrum management [9].

The deployment of REM's will lead to better understanding of the radio environment by the licensed and unlicensed users, realization of dynamic spectrum access and overall improvement of the network performance[10]. This paper is divided as follows; section II presents an overview of REM, in section III the architecture of REM is discussed. REM construction techniques are presented in section IV, section $\mathrm{V}$ discusses propagation techniques used in REM construction. Finally section VI highlights the quality metrics used in determining the accuracy of these maps. 
II.

\section{REM OVERVIEW}

Theoretically, CR could be able to detect the presence of spectrum hole through spectrum sensing thus making it possible for an unlicensed user to utilize the radio channel [11]. However, due to more work being needed on CR's capability and accuracy most regulators (OFCOM, FCC, ECC etc.) have adopted the use of Television White Space (TVWS) geo-location database approach for dynamic spectrum access in the terrestrial TV broadcast services band i.e. the VHF and UHF frequency bands. According to [12], "The main difference of REM from the geo-location DB is that REM generates spectrum map by processing the data collected from multiple sources (e.g. measurements from MCDs and regulations from the regulatory bodies in a country) with its cognitive engine, and therefore can easily adapt to dynamic operating environments whereas geo-location DB stores quasi-static information". REM therefore makes nodes in a given network environmentally aware of their surroundings thus turning them into intelligent nodes that make informed decisions bringing the concept of CR closer to reality. Figure 1 presents a holistic overview of the various aspects of REM from geo-location database, architecture, data model, quality metrics, construction methods and challenges encountered in REM construction and research.

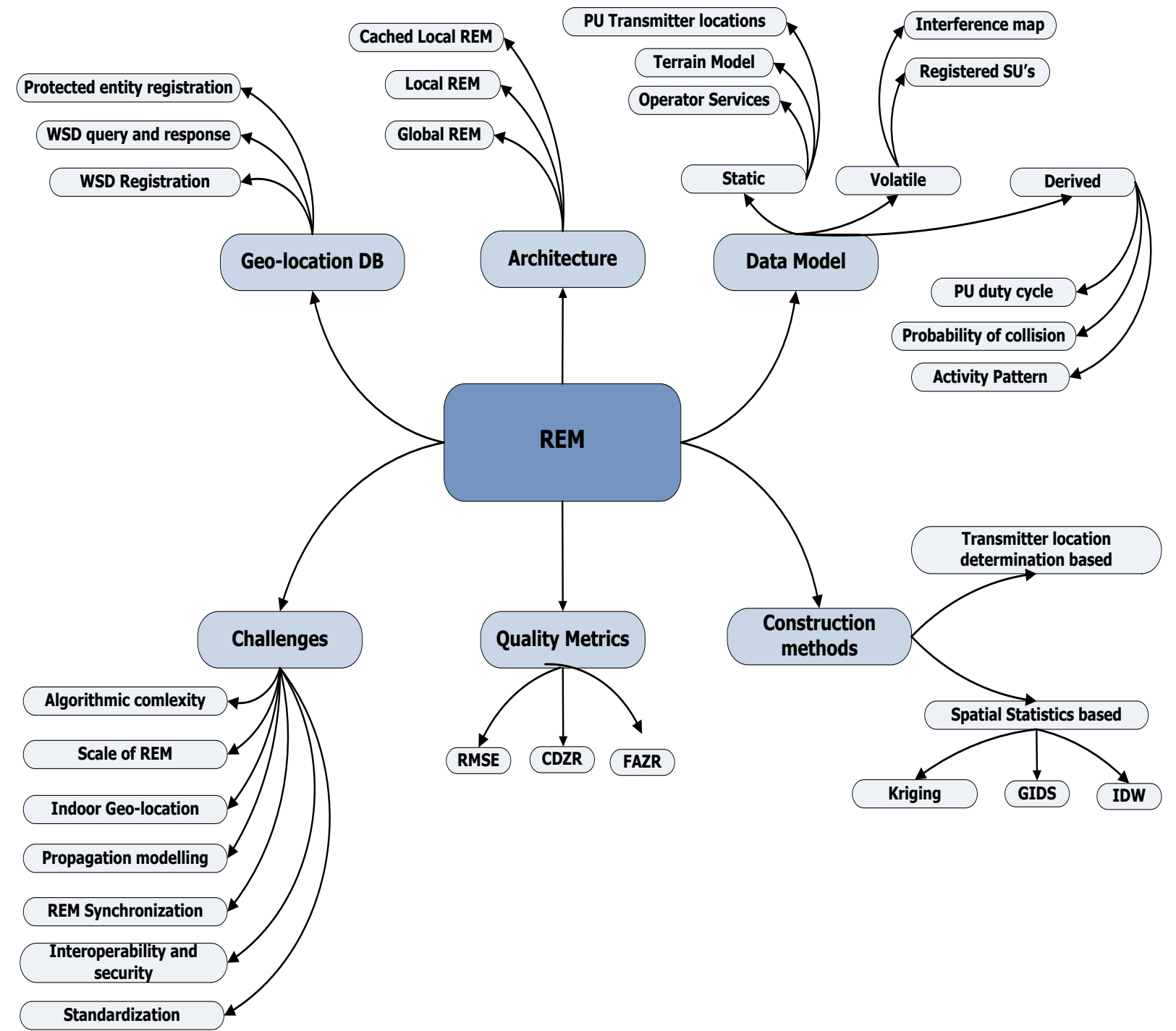

Fig 1 REM Components [12]

III.

\section{REM ARCHITECTURE}

A CR generally has two kinds of REM's i.e. local and Global REM. Global REM provides extensive processing capabilities while local REM increases the responsiveness of the system [13]. The scale of the global REM depends on the size of network's service area whereas the scale of local REM is determined by the unit service area, e.g., a cell for a cellular network. It could be then said that the space complexity of REM depends on the number of nodes, number of channels, size of network coverage, granularity of REM information, etc. [8]. 
Synchronization of REM's is very important for the proper operation in terms of accuracy and reliability of the information being provided. It is therefore paramount that both local and global REM's synchronize so as to update their observations and experiences Furthermore, since the spectrum policies and regulations vary from country to country, each country must therefore have its own map that will be in harmony with its neighbors especially in the border areas [12].

\section{REM CONSTRUCTION METHODS}

As REM is required to provide reliable information in order to meet the stringent criteria of no-harmful interference for the licensed user, it must then be constructed in a timely manner to capture the changing dynamics of the CRN. For the purpose of this section, we shall limit the concept of REM on deriving interference level of the CRN. Based on literature, REM construction methods could be broadly classified into three methods: spatial statistics based approaches (direct approach), transmitter location determination based methods (indirect approach) and hybrid approach that involves both direct and indirect methods [13].

\section{A. Spatial Statistics Based Methods}

In [1], the task of obtaining radio environment information could be formulated as:" Given a finite number of localized radio measurement, how can we deduce the missing values of the measured quantity (the value of total received signal power on the considered frequency band) on the whole area of interest, with a given reliability degree?" The basic solution to this problem as presented in [14] consists of choosing interpolation technique that will generate "sufficiently" precise estimation of the real value of the considered quantity. The term "sufficiently" precise (or estimated value which minimizes some reliability metric representing the interpolation error) denotes that the obtained value generates a reasonable spectrum occupancy information, thus in the same time justifying the limited number of measurements performed.

In spatial statistics approach, spatial maps are developed using data obtained from radio measurements for a given location. Since it's impossible to obtain measurements for all locations in a given area, points without any measurement can then be estimated as a function of measured data as indicated in figure 2. Well known spatial estimation techniques include Kriging, Inverse Distance Weighted (IDW), and nearest neighbor interpolation techniques [12]

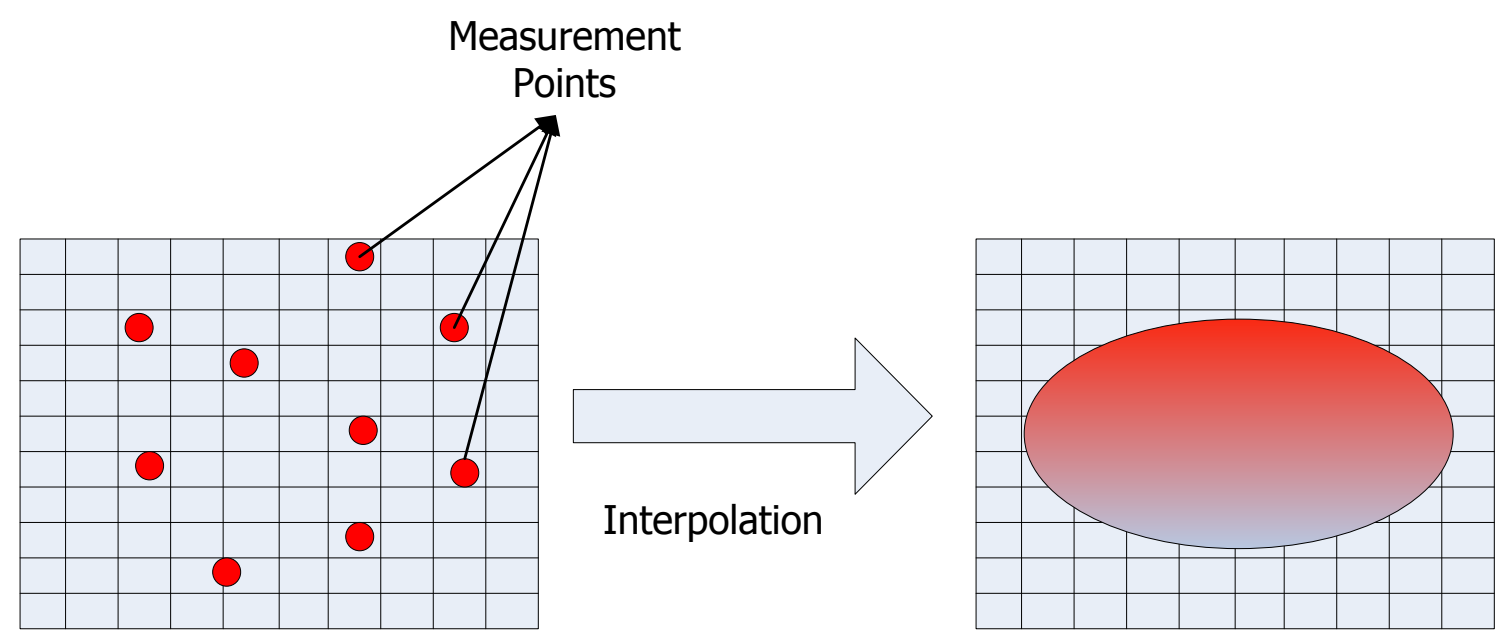

Fig. 2 Spatial Statistics based approach [15]

In kriging interpolation method which is a minimum mean squared error method of spatial prediction that depends upon the second order properties of the process [16]. The aim is to estimate unknown values at a given location $\mathrm{X}_{0}$ from the data obtained during the radio measurements. The estimation is achieved by assigning a weight factor $\omega_{\mathrm{i}}(\mathrm{i}=1, \ldots, \mathrm{N})$ to each value measured by the $\mathrm{N}$ nodes, as follows:

$$
\widehat{P}\left(x_{0}\right)=\sum_{i=1}^{N} \omega_{i} P\left(x_{i}\right)
$$

Where $\widehat{\mathrm{P}}\left(\mathrm{x}_{0}\right)$ is the estimated/interpolated value. The weights are determined by minimizing the error variance of the general Kriging linear estimator [17, 18]. In [19] a prototype for developing REM's from massive data set with linear 
computational complexity was developed using fixed rank Kriging which was first demonstrated in [20, 21], to enable processing of tens or hundreds of thousands of measurements on a typical laptop in an interactive manner. REM's were constructed in [22] from data obtained in Caracas Venezuela. The work employed three interpolation techniques: Kriging, IDW and SPLINE where they found out that IDW performed better overall. [1] introduced interference cartography with the aim of detecting spectrum hole for dynamic spectrum access. Kriging technique was applied to interference data obtained from a radio network simulator; the researchers found out that the developed REM could enhance the performance of secondary spectrum usage.

An REM was developed in the $2.5 \mathrm{GHz}$ WiMax network at the University of Colorado. It was found that this approach to radio environment mapping is feasible and produces maps that are more accurate and informative than both explicitly tuned path loss models and basic data fitting approaches.

IDW uses the weights inversely proportional to the distance between $p$ and $p_{i}$ for weighting $z_{i}$ values and evaluates the weighted sum of measured values for calculating $\hat{z}(p)$. Given,

$$
w_{i}(p)=d\left(p, p_{i}\right)^{-\beta}
$$

Where $\beta$ is the IDW power, $d(.,$.$) is the distance function, w_{i}(p)$ is the weight of $z_{i}$ for evaluating estimate value at point $\mathrm{p}$. For $\beta=1$ the method is known as IDW1 while for $\beta=2$ its known as IDW2 [12]. For other interpolation techniques refer to table 1 .

Table I Summary of REM Interpolation Techniques

\begin{tabular}{|c|l|l|}
\hline S/N & \multicolumn{1}{|c|}{ Method } & \multicolumn{1}{|c|}{ Description } \\
\hline 1 & Kriging[1] & $\begin{array}{l}\text { It is a linear spatial interpolation method; it assumes that the } \\
\text { missing values could be estimated by weighted linear combinations } \\
\text { of the available neighboring values. The weights are computed } \\
\text { based on the measured values expressed through the spatial auto } \\
\text { covariance function. }\end{array}$ \\
\hline 2 & Nearest Neighbor [23] & $\begin{array}{l}\text { It's the simplest method to implement. The missing values are } \\
\text { obtained as the value measured by the closest sensor (which } \\
\text { corresponds to the sensor with the shortest distance). }\end{array}$ \\
\hline 3 & $\begin{array}{l}\text { Inverse Distance Weight } \\
{[24]}\end{array}$ & $\begin{array}{l}\text { It's based on the concept that nearby values contribute more to the } \\
\text { estimated values than distant values. The influence of a measured } \\
\text { data point is inversely related to the distance from the unknown } \\
\text { location that is being measured. }\end{array}$ \\
\hline
\end{tabular}

\section{B. Transmitter Estimation Based Method}

Estimating the location of transmitters in a given network has several applications of interest to researchers. Different received signal parameters like Time of Arrival (TOA), Angle of Arrival (AOA), and Received signal Strength (RSS) can be utilized for this purpose [25].

For example, the angle of the transmitter can be obtained from AOA of a signal at the receiver. Estimating the flight time of a signal can also be obtained from TOA which also provides information about the distance from a transmitter to a receiver [26].

The spatial statistical based methods directly approximate the signal strength without taking into consideration the transmitters which are the sources of the power. Information on the transmitter location can ease the REM construction process. In transmitter location based methods, the first stage involves localizing the transmitter and deriving its properties.

The estimated signal strength at each location by using propagation modeling is then done. This method has been found to have more degrees of freedom: multiple transmitters, transmitter properties such as antenna propagation pattern, and accurate characterization of the propagation environment [12]. A summary of three forms of transmitter estimated based methods are provided in table 2 . 


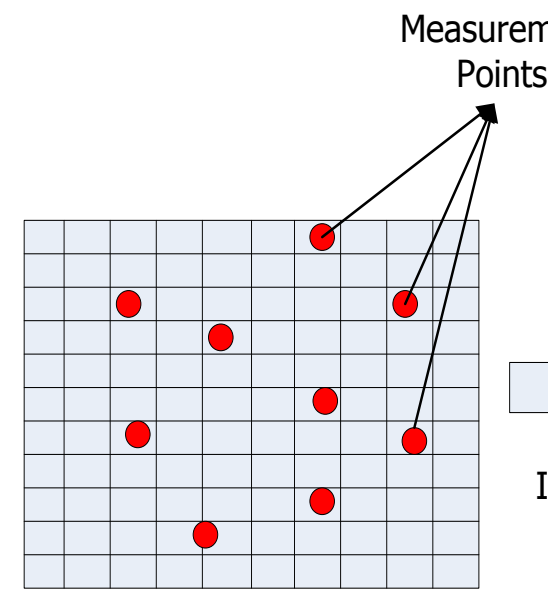

RF REM data

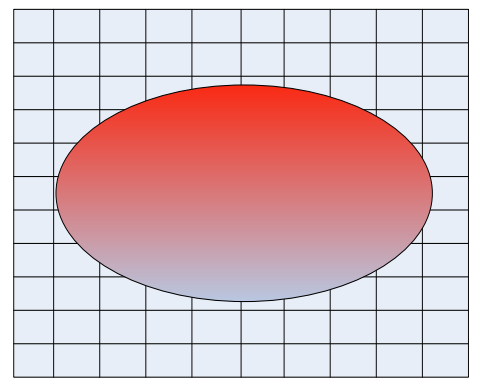

Fig. 3 Transmitter estimation based approach [15]

\section{Tx estimated} location

Interpolation

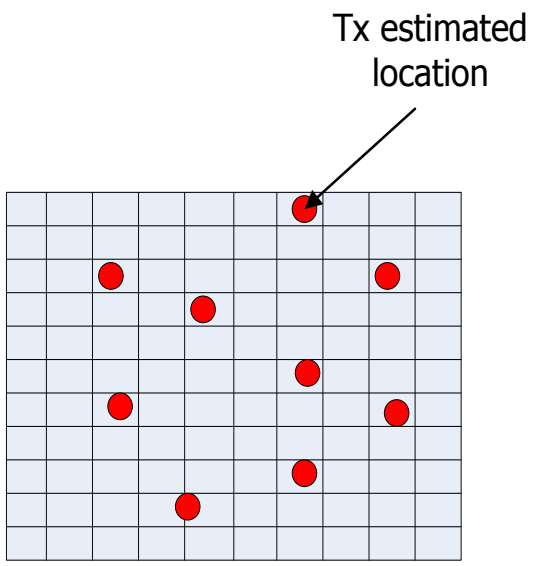

\author{
RF coverage \\ calculation using \\ radio propagation \\ model
}

\title{
C. Hybrid Methods
}

These are a combination of techniques used in both spatial interpolation based methods and transmitter estimation based methods. This shows that the number of possible solutions can be numerous. For example, having chosen the interpolation method of measurement results, a REM layer is created, finally based on the processing of this image, the propagation and transmission parameters are estimated [15].

\section{PROPAGATION MODELS}

Studies on propagation models dated back to the early 1940's; over the years several models have been developed. Due to their diversity in terms of factors affecting the environment on the EM wave propagation losses, seven categories and fourteen subcategories were proposed in [28].Broadly speaking, most models could be classified into deterministic, stochastic and mixed models.

According to [15], propagation loss can be decomposed into components that constitute the different propagation path factors

$$
\mathrm{L}_{\mathrm{p}}=\mathrm{L}_{\mathrm{fsd}}+\mathrm{L}_{\mathrm{d}}+\mathrm{L}_{\mathrm{sp}}+\mathrm{L}_{\mathrm{g}}+\mathrm{L}_{\mathrm{r}}+\sum \mathrm{L}_{\mathrm{oi}}+\mathrm{L}_{\mathrm{m}}
$$

Where $\mathrm{L}_{\mathrm{fsd}}$ is free space propagation losses, $\mathrm{L}_{\mathrm{d}}$ is the propagation losses related to diffraction, $\mathrm{L}_{\mathrm{sp}}$ are losses related to route geometry, $\mathrm{L}_{\mathrm{g}}$ are the atmospheric gas losses, $\mathrm{L}_{\mathrm{r}}$ are losses associated with atmospheric precipitation, $\mathrm{L}_{\mathrm{oi}}$ are losses introduced by various terrain obstacles such as type of buildings, wooded area etc., $\mathrm{L}_{\mathrm{m}}$ are additional losses introduced by model's authors, e.g. tropospheric scattering, ionospheric reflections etc.

Several propagation models has been implemented in the ATDI (ICS Telecom, HTZ Warfare) software tools for planning and simulation of wireless systems [29]. This software can be used to select the propagation model that is most adequate the designers requirements. Table 1 shows the comparison of accuracy of the four propagation models: 
Vol. 8, Issue 4, April 2019

1. Deterministic ITU-R 525 Basic, Diffraction method- Deygout 94, Subpaths - fine integration

2. Stochastic ITU-R 1812-3, (50\% T, 50\%L)

3. Okumura-Hata original

4. Longley-Rice ITM/NTIA $(50 \% \mathrm{~T}, 50 \% \mathrm{~L})$

Four parameters were used to evaluate the propagation model accuracy: standard deviation and average error of differences between measured and calculated values, correlation of these values, and percentage of results with differences less than $6 \mathrm{~dB}[15]$.

Table III Accuracy Parameters of Choosen Propagation Models

\begin{tabular}{|c|c|c|c|c|c|}
\hline Model & $\begin{array}{c}\text { Standard } \\
\text { Deviation }(\mathrm{dB})\end{array}$ & $\begin{array}{c}\text { Average } \\
\text { Error }(\mathrm{dB})\end{array}$ & $\begin{array}{c}\text { Correlation } \\
\text { Factor }\end{array}$ & Differences<6dB\% & Symmetry \\
\hline 1 & 3.42 & 0.84 & 0.97 & 91.17 & YES \\
\hline 2 & 3.62 & 1.57 & 0.97 & 88.55 & YES \\
\hline 3 & 5.10 & 1.38 & 0.94 & 83.46 & NO \\
\hline 4 & 6.28 & 2.12 & 0.94 & 78.75 & YES \\
\hline
\end{tabular}

VI. REM QUALITY

Data obtained from radio measurement constitute the essential component of REM construction. However, estimated REM and actual REM may differ at some locations. The accuracy of these REMs are determined by the quality metrics. These quality metrics can be computed by comparing estimated REM with true REM in terms of correct detection zone, false alarm zone, and Root Mean Square Error (RMSE). For detailed explanation on REM quality metrics, refer to [12]

\section{CONCLUSION}

REM is a crucial component in the quest to realizing dynamic access especially in the TVWS band thus enabling the concept to cognitive radio to come to reality. The importance of REM cannot be overemphasized as it is the central block that contains vital network information such as physical network information, policies, and licensed user activity pattern amongst others. In this work, a general overview which is aimed at serving as a guide in constructing REM is present. The research highlighted the main areas that central to REM construction these include: the architecture of REM, construction methods that are basically divided into three namely; spatial interpolation based methods, transmitter location based methods and finally hybrid methods. Furthermore, an overview of the propagation models generally used in REM construction is presented with emphasis the accuracy parameters of these models being highlighted. Finally, quality metrics used to determine the accuracy of constructed REMs is presented.

\section{REFERENCES}

[1]. Alaya-Feki, Afef Ben Hadj, et al. "Informed spectrum usage in cognitive radio networks: Interference cartography." 2008 IEEE 19 th International Symposium on Personal, Indoor and Mobile Radio Communications. IEEE, 2008.

[2]. Haykin, Simon. "Cognitive radio: brain-empowered wireless communications." IEEE journal on selected areas in communications 23.2 (2005): 201-220.

[3]. Najashi, Barau Gafai, Feng Wenjiang, and Mohammed Dikko Almustapha. "Spectrum hole prediction based on historical data: A neural network approach." arXiv preprint arXiv:1401.0886 (2014).

[4]. Pagadarai, Srikanth, and Alexander M. Wyglinski. "A quantitative assessment of wireless spectrum measurements for dynamic spectrum access." 2009 4th International Conference on Cognitive Radio Oriented Wireless Networks and Communications. IEEE, 2009.

[5]. Han, Yanfeng, et al. "Spectrum occupancy measurement: Focus on the TV frequency." 2010 2nd International Conference on Signal Processing Systems. Vol. 2. IEEE, 2010.

[6]. Holland, Oliver, et al. "Spectrum power measurements in $2 \mathrm{G}$ and $3 \mathrm{G}$ cellular phone bands during the 2006 football world cup in germany." 2007 2nd IEEE International Symposium on New Frontiers in Dynamic Spectrum Access Networks. IEEE, 2007.

[7]. Alaya-Feki, Afef Ben Hadj, Eric Moulines, and Emmanuelle Villebrun. "Exploiting radio measurements in wireless mobile networks with advanced signal processing." 2007 Third International Conference on Wireless and Mobile Communications (ICWMC'07). IEEE, 2007.

[8]. Zhao, Youping. Enabling cognitive radios through radio environment maps. Diss. Virginia Tech, 2007.

[9]. Faint, Stephanie, Oktay Üreten, and Trida Willink. "Impact of the number of sensors on the network cost and accuracy of the radio environment map." CCECE 2010. IEEE, 2010.

[10]. Li, Jingming, et al. "Recent Advances in Radio Environment Map: A Survey." International Conference on Machine Learning and Intelligent Communications. Springer, Cham, 2017.

[11]. Fanan, A., et al. "Implementation of combined geolocation database and infrastructure sensing in TV bands using different spectrum devices." 2017 25th Telecommunication Forum (TELFOR). IEEE, 2017.

[12]. Yilmaz, H. Birkan, et al. "Radio environment map as enabler for practical cognitive radio networks." IEEE Communications Magazine 51.12 (2013): 162-169.

[13]. Mahapatra, Rajarshi, and Emilio Calvanese Strinati. "Interference-aware dynamic spectrum access in cognitive radio network." 2011 IEEE 22nd International Symposium on Personal, Indoor and Mobile Radio Communications. IEEE, 2011. 


\section{International Journal of Advanced Research in Computer and Communication Engineering}

Vol. 8, Issue 4, April 2019

[14]. Angjelicinoski, Marko, Vladimir Atanasovski, and Liljana Gavrilovska. "Comparative analysis of spatial interpolation methods for creating radio environment maps." 2011 19thTelecommunications Forum (TELFOR) Proceedings of Papers. IEEE, 2011.

[15]. Gajewski, Piotr. "Propagation models in radio environment map design." 2018 Baltic URSI Symposium (URSI). IEEE, 2018.

[16]. Cressie, Noel. "Statistics for spatial data." Terra Nova 4.5 (1992): 613-617.

[17]. E. H. Isaaks and R. M. Srivastava, An Introduction to Applied Geostatistics, Oxford University Press, New York, 1989.

[18]. Goovaerts, Pierre. Geostatistics for natural resources evaluation. Oxford University Press on Demand, 1997.

[19]. Riihijärvi, Janne, Jad Nasreddine, and Petri Mähönen. "Demonstrating radio environment map construction from massive data sets." 2012 IEEE International Symposium on Dynamic Spectrum Access Networks. IEEE, 2012.

[20]. Cressie, Noel, and Gardar Johannesson. "Fixed rank kriging for very large spatial data sets." Journal of the Royal Statistical Society: Series B (Statistical Methodology) 70.1 (2008): 209-226.

[21]. Cressie, Noel, Tao Shi, and Emily L. Kang. "Fixed rank filtering for spatio-temporal data." Journal of Computational and Graphical Statistics 19.3 (2010): 724-745

[22]. Azpurua, Marco, and K. Dos Ramos. "A comparison of spatial interpolation methods for estimation of average electromagnetic field magnitude." Progress In Electromagnetics Research M 14.2010 (2010): 135-145.

[23]. Bolea, Liliana, Jordi Pérez-Romero, and Ramon Agustí. "Received signal interpolation for context discovery in Cognitive Radio." 2011 The 14th International Symposium on Wireless Personal Multimedia Communications (WPMC). IEEE, 2011.

[24]. Azpurua, Marco, and K. Dos Ramos. "A comparison of spatial interpolation methods for estimation of average electromagnetic field magnitude." Progress In Electromagnetics Research M 14.2010 (2010): 135-145.

[25]. Gezici, Sinan. "A survey on wireless position estimation." Wireless personal communications 44.3 (2008): 263-282.

[26]. Ozsahin, Mustafa Tugrul, and Tuna Tugcu. "Transmitter location estimation for radio environment map construction using software defined radio." 2014 IEEE 21st Symposium on Communications and Vehicular Technology in the Benelux (SCVT). IEEE, 2014.

[27]. Pesko, Marko, et al. "Radio environment maps: The survey of construction methods." KSII Transactions on Internet \& Information Systems 8.11 (2014).

[28]. Phillips, Caleb, Douglas Sicker, and Dirk Grunwald. "A survey of wireless path loss prediction and coverage mapping methods." IEEE Communications Surveys \& Tutorials 15.1 (2013): 255-270

[29]. Radio Propagation in ATDI Tools, http://www.atdi.com/doccenter/ics\%20telecom\%20-\%20propagation\%20models\%20us.pdf 